\title{
MAXIMUM PRINCIPLES FOR PARABOLIC SYSTEMS COUPLED IN BOTH FIRST-ORDER AND ZERO-ORDER TERMS
}

\author{
CHIPING ZHOU \\ Department of Mathematics \\ University of Hawaii-HCC \\ 874 Dillingham Boulevard \\ Honolulu, HI 96817
}

(Received October 21, 1992 and in revised form June 30, 1993)

\begin{abstract}
Some generalized maximum principles are established for linear second-order parabolic systems in which both first-order and zero-order terms are coupled.
\end{abstract}

KEY WORDS AND PHRASES. Maximum principles, parabolic systems, strongly coupled, complex-valued.

1991 AMS SUBJECT CLASSIFICATION CODES. 35B50, 35K40.

\section{INTRODUCTION.}

Hile and Protter [2] proved that the Euclidean length of the solution vector $u \in C^{2}(D) \cap C(\bar{D})$ of the second-order elliptic system

$$
\sum_{i, k=1}^{n} a_{\imath k}(x) \frac{\partial^{2} u_{s}}{\partial x_{\imath} \partial x_{k}}+\sum_{i=1}^{n} \sum_{j=1}^{m} b_{s \imath \jmath}(x) \frac{\partial u_{\jmath}}{\partial x_{i}}+\sum_{j=1}^{m} c_{s \jmath}(x) u_{\jmath}=0, \quad s=1, \cdots, m,
$$

can be bounded by a constant times the maximum of its boundary values under a "small" condition which requires that either the domain $D$ or the coefficients $b_{s i j}$ and $c_{s j}$ are sufficiently small. In this paper, we have established the same kind of maximum principle for the secondorder parabolic system

$$
\sum_{i, k=1}^{n} a_{\imath k}(x, t) \frac{\partial^{2} u_{s}}{\partial x_{\imath} \partial x_{k}}-\frac{\partial u_{s}}{\partial t}+\sum_{i=1}^{n} \sum_{j=1}^{m} b_{s \imath}(x, t) \frac{\partial u_{\jmath}}{\partial x_{\imath}}+\sum_{j=1}^{m} c_{s \jmath}(x, t) u_{\jmath}=0,1 \leq s \leq m .
$$

Moreover, our parabolic version of the maximum principle holds without any "small" conditions.

When the coupling occurs only in the zero-order terms (i.e., in the case of $b_{s i j}=0$ for all $i, j, s$ except when $j=s$ ), the above systems are called weakly coupled systems. For weakly coupled second-order parabolic systems, similar maximum principles have been obtained by Stys [4] and Zhou [6]. Under different assumptions, different maximum principles in which the components rather than the Euclidean length of the solution vector are bounded can be found in Protter and Weinberger [3] and Dow [1]. In Weinberger's paper [5], both kinds of maximum principles have been reformulated and studied in terms of invariant sets.

\section{MAIN RESULTS.}

Consider a second-order parabolic operator with real coefficients,

$$
M \equiv \sum_{i, k=1}^{n} a_{\imath k}(x, t) \frac{\partial^{2}}{\partial x_{\imath} \partial x_{k}}-\frac{\partial}{\partial t}, \quad a_{\mathfrak{\imath}}=a_{\mathfrak{\jmath}}
$$

in a general bounded domain $\Omega$ in $\mathbb{R}^{n} \times \mathbb{R}_{t}(n \geq 1)$ with the boundary $\partial \Omega:=\partial_{p} \Omega \cup \partial_{t} \Omega$. Here $\partial_{p} \Omega$ is the parabolic boundary of $\Omega$ and $\partial_{t} \Omega$ : $=\partial \Omega \backslash \partial_{p} \Omega$. We suppose that $\Omega \subset D \times(0, T)$ where $D$ is a 
bounded domain in $\mathbb{R}^{n}$ and $0<T<\infty$. The operator $M$ is assumed to be uniformly parabolic in $\Omega$; i.e., there is a constant $\delta>0$ such that for all $(x, t) \in \Omega$ and all $\left(y_{1}, \cdots, y_{n}\right)$ in $\mathbb{C}^{n}$ the inequality

$$
\sum_{\imath, k=1}^{n} a_{\mathfrak{i} k}(x, t) y_{\imath} \bar{y}_{k} \geq \delta \sum_{i=1}^{n}\left|y_{\mathbf{i}}\right|^{2}
$$

holds. The operator $M$ is the principal part of each equation in the second-order parabolic system

$$
M u_{s}+\sum_{i=1}^{n} \sum_{j=1}^{m} b_{s i j}(x, t) \frac{\partial u_{\jmath}}{\partial x_{\imath}}+\sum_{j=1}^{m} c_{s \jmath}(x, t) u_{\jmath}=0, \quad s=1,2, \cdots, m .
$$

We suppose that the complex-valued coefficients $b_{s i j}, c_{s j}$ have the property that for all $\xi \in \mathbf{C}^{m}$ and all $(x, t) \in \Omega$.

$$
r, s=1\left[c_{s r}+\bar{c}_{r s}+\frac{1}{2} \sum_{j=1}^{m} \sum_{k, i=1}^{n} A_{k i} b_{s i j} \bar{b}_{r k s}\right] \xi_{r} \bar{\xi}_{s} \leq K|\xi|^{2}, \text { for some } K>0 .
$$

Here $\left(A_{k z}\right)=\left(A_{i k}\right)$ denotes the inverse matrix of $\left(a_{t k}\right)$. A solution $u=\left(u_{1}, u_{2}, \cdots, u_{m}\right)$ is a complex-valued $C^{2,1}\left(\Omega \cup \partial_{t} \Omega\right) \cap C(\bar{\Omega})$ function which satisfies $(2)$ in $\Omega$. Here $C^{k, h}(\Omega)$ is defined as the set of functions $f(x, t)$ having all $x$ (space) derivatives of order $\leq k$ and $t$ (time) derivatives of order $\leq h$ continuous in $\Omega$.

THEOREM 1. Assume conditions (1.1) and (1.3) hold. If $u$ is a solution of (2.2) and $\alpha$ is a positive $C^{2,1}\left(\Omega \cup \partial_{t} \Omega\right)$ function, then the product $\alpha|u|^{2}=\alpha \sum_{j=1}^{m}\left|u_{\jmath}\right|^{2}$ cannot attain a positive maximum at any point in $\Omega \cup \partial_{t} \Omega$ where $\alpha$ satisfies

$$
\alpha^{-1} M \alpha-2 \alpha^{-2} \sum_{i, k=1}^{n} a_{i k} \frac{\partial \alpha}{\partial x_{i}} \frac{\partial \alpha}{\partial x_{k}}>K .
$$

PROOF. We set $p=|u|^{2}=\sum_{s=1}^{m}\left|u_{s}\right|^{2}$ and find

$$
M(\alpha p)=p M \alpha+\alpha M p+2 \sum_{i, k=1}^{n} a_{i k} \frac{\partial \alpha}{\partial x_{i}} \frac{\partial p}{\partial x_{k}} .
$$

At a point $(x, t) \in \Omega \cup \partial_{t} \Omega$ where $\alpha p$ attains a maximum, we have

and (2.5) becomes

$$
0 \leq \frac{\partial(\alpha p)}{\partial t}, \quad 0=\frac{\partial(\alpha p)}{\partial x_{k}}=\alpha \frac{\partial p}{\partial x_{k}}+p \frac{\partial \alpha}{\partial x_{k}}, \quad 1 \leq k \leq n,
$$

A direct computation yields

$$
\underset{\text { yields }}{M(\alpha p)}=p\left[M \alpha-2 \alpha^{-1} \sum_{i, k=1}^{n} a_{\mathfrak{i} k} \frac{\partial \alpha}{\partial x_{\imath}} \frac{\partial \alpha}{\partial x_{k}}\right]+\alpha M p
$$

$$
\begin{aligned}
& M p=\sum_{s=1}^{m}\left[u_{s} M \bar{u}_{s}+\bar{u}_{s} M u_{s}+2 \sum_{i, k=1}^{n} a_{i k} \frac{\partial u_{s}}{\partial x_{s}} \frac{\partial \bar{u}_{s}}{\partial x_{k}}\right] \\
& =\sum_{s=1}^{m}\left\{-2 \operatorname{Re}\left[\bar{u},\left\{\sum_{i=1}^{n} \sum_{j=1}^{m} b_{s i j} \frac{\partial u_{j}}{\partial x_{i}}+\sum_{j=1}^{m} c_{s,} u,\right)\right]+2 \sum_{i, k=1}^{n} a_{i k} \frac{\partial u_{s}}{\partial x_{i}} \frac{\partial \bar{u}_{s}}{\partial x_{k}}\right\} \\
& =2\left\{\sum_{j=1}^{m} \sum_{i, k=1}^{n} a_{i k}\left[\frac{\partial u_{j}}{\partial x_{i}}-\frac{1}{2} \sum_{q=1}^{n} \sum_{r=1}^{m} A_{s q} \bar{b}_{r q j} u_{r}\right]\left[\frac{\partial \bar{u}_{j}}{\partial x_{k}}-\frac{1}{2} \sum_{q=1}^{n} \sum_{s=1}^{m} A_{k q} b_{s q} \bar{u} \cdot\right]\right. \\
& \left.-\frac{1}{4} \sum_{r, s=1}^{m}\left[\sum_{k, q=1}^{n} \sum_{j=1}^{m} A_{k q} b_{s q j} \bar{b}_{r k j}\right] u_{r} \bar{u}_{s}\right\}-\sum_{r, s=1}^{m}\left(c_{s r}+\bar{c}_{s r}\right) u_{r} \bar{u}, \\
& \geq-K \sum_{s=1}^{m}\left|u_{s}\right|^{2}=-K p .
\end{aligned}
$$


Hence, from (2.6), we have

$$
M(\alpha p) \geq \alpha p\left[\alpha^{-1} M \alpha-2 \alpha^{-2} \sum_{\imath, k=1}^{n} a_{\imath k} \frac{\partial \alpha}{\partial x_{\imath}} \frac{\partial \alpha}{\partial x_{k}}-K\right] .
$$

This inequality holds at any point in $\Omega \cup \partial_{t} \Omega$ where $\alpha p$ attains a maximum. Thus $\alpha p$ cannot achieve a positive maximum at any point in $\Omega \cup \partial_{t} \Omega$ where the quantity in brackets in (2.7) is positive. The theorem is established.

REMARK. If for all $(x, t) \in \Omega$,

$$
\left|c_{s \jmath}\right| \leq K_{0},\left|b_{s i}\right| \leq K_{1}, 1 \leq i \leq n, 1 \leq j, s \leq m \text {, for some } K_{0}, K_{1} \in \mathbb{R},
$$

then for any $\xi \in \mathbb{C}^{m}$,

$$
\begin{aligned}
\sum_{r, s=1}^{m} & {\left[c_{s r}+\bar{c}_{r s}+\frac{1}{2} \sum_{j=1}^{m} \sum_{k, i=1}^{n} A_{k_{s}} b_{s t} \bar{b}_{r k s}\right] \xi_{r} \bar{\xi}_{s} } \\
& \leq \sum_{r, s=1}^{m}\left|c_{s r}\right|\left(\left|\xi_{r}\right|^{2}+\left|\xi_{s}\right|^{2}\right)+\frac{1}{2} \sum_{j=1}^{m} \sum_{k, i=1}^{n} A_{k s}\left(\sum_{s=1}^{m} b_{s i} \bar{\xi}_{s}\right)\left(\sum_{r=1}^{m} \bar{b}_{r k s} \xi_{r}\right) \\
& \leq 2 m K_{0} \sum_{s=1}^{m}\left|\xi_{s}\right|^{2}+\frac{1}{2 \delta} \sum_{j=1}^{m} \sum_{\imath=1}^{n}\left|\sum_{s=1}^{m} b_{s i j} \bar{\xi}_{s}\right|^{2} \\
& \leq 2 m K_{0}|\xi|^{2}+\frac{m}{2 \delta} \sum_{j=1}^{m} \sum_{i=1}^{n} \sum_{s=1}^{m}\left|b_{s i j} \bar{\xi}_{s}\right|^{2} \leq\left[2 m K_{0}+(2 \delta)^{-1} n m^{2} K_{1}^{2}\right]|\xi|^{2},
\end{aligned}
$$

which is the condition (2.3) with $K:=2 m K_{0}+(2 \delta)^{-1} n m^{2} K_{1}^{2}$. Hence, the single bound (2.3) in Theorem 1 can be replaced by the separate bounds $(2.8)$ with $K:=2 m K_{0}+(2 \delta)^{-1} n m^{2} K_{1}^{2}$.

Under the conditions (2.1) and (2.3) (or (2.1) and (2.8)), by choosing $\alpha(x, t)=e^{-(K+\varepsilon) t}, \varepsilon>0$, the condition (2.4) will be satisfied. Hence from Theorem 1, we get the following maximum principle:

COROLLARY 2 (Maximum Principle). For any solution $u$ of the system (2.2), the function

$$
|u(x, t)|^{2} \exp [-(K+\varepsilon) t], \varepsilon>0,
$$

does not attain a positive maximum in $\Omega \cup \partial_{t} \Omega$, and

$$
\|u\|_{0, \Omega} \leq \exp (K T / 2)\|u\|_{0, \partial_{p} \Omega} .
$$

Here $K=(2 \delta)^{-1} n m^{2} K_{1}^{2}+2 m K_{0}$ and $\|u\|_{0, \Omega}:=\underset{(x, t) \in \Omega}{\sup }|u(x, t)|$.

REMARK. Results similar to Theorem 1 and Corollary 2 for second-order elliptic systems were proven by Hile and Protter [2] (under a condition which is similar to (2.8)). But their maximum principle for elliptic systems only holds under the restriction that either the domain $D$ is sufficiently small or the coefficients of the elliptic system are restricted sufficiently. Corollary 2 tells us that these restrictions can be lifted for parabolic systems.

COROLLARY 3 (Uniqueness). The system (2.2) with the initial-boundary condition

$$
\left.u\right|_{\partial_{p} \Omega}=\varphi(x, t)
$$

has at most one solution $u \in C^{2,1}(\Omega \cup \Omega) \cap C(\bar{\Omega})$.

Theorem 1 can be used to obtain bounds on the gradient of the $C^{3,2}$ solution of the parabolic system (2.2), provided the coefficients are $C^{1}$ and

$$
\left\|a_{\imath k}\right\|_{1, \Omega} \leq L_{2},\left\|b_{s i}\right\|_{1, \Omega} \leq L_{1},\left\|c_{s \jmath}\right\|_{1, \Omega} \leq L_{0}, \text { for some } L_{2}, L_{1}, L_{0} \in \mathbb{R} .
$$

Here $\|f\|_{1, \Omega}:=\|f\|_{0, \Omega}+\sum_{i=1}^{n}\left\|\frac{\partial f}{\partial x_{i}}\right\|_{0, \Omega}+\left\|\frac{\partial f}{\partial t}\right\|_{0, \Omega}$.

We differentiate (2.2) with respect to $x_{h}$ and $t$, and get $m(n+1)$ equations: 


$$
\begin{aligned}
& M\left(\frac{\partial u_{s}}{\partial x_{h}}\right)+\sum_{i, k=1}^{n} \frac{\partial a_{i k}}{\partial x_{h}} \frac{\partial}{\partial x_{i}}\left(\frac{\partial u_{s}}{\partial x_{h}}\right)+\sum_{i=1}^{n} \sum_{j=1}^{m} b_{s i}, \frac{\partial}{\partial x_{i}}\left(\frac{\partial u_{j}}{\partial x_{h}}\right) \\
& +\sum_{i=1}^{n} \sum_{j=1}^{m} \frac{\partial b_{s i \jmath}}{\partial x_{h}} \frac{\partial u_{\jmath}}{\partial x_{\imath}}+\sum_{j=1}^{m} c_{s \jmath} \frac{\partial u_{\jmath}}{\partial x_{h}}+\sum_{j=1}^{m} \frac{\partial c_{s \jmath}}{\partial x_{h}} u_{\jmath}=0 \\
& s=1,2, \cdots, m \text { and } h=1,2, \cdots, n \text {; } \\
& M\left(\frac{\partial u_{s}}{\partial t}\right)+\sum_{i, k=1}^{n} \frac{\partial a_{i k}}{\partial t} \frac{\partial}{\partial x_{i}}\left(\frac{\partial u_{s}}{\partial x_{k}}\right)+\sum_{i=1}^{n} \sum_{j=1}^{m} b_{s i} \frac{\partial}{\partial x_{i}}\left(\frac{\partial u_{j}}{\partial t}\right) \\
& +\sum_{i=1}^{n} \sum_{j=1}^{m} \frac{\partial b_{s i \jmath}}{\partial t} \frac{\partial u_{\jmath}}{\partial x_{i}}+\sum_{j=1}^{m} c_{s j} \frac{\partial u_{\jmath}}{\partial t}+\sum_{j=1}^{m} \frac{\partial c_{s \jmath}}{\partial t} u_{\jmath}=0 \\
& h=1,2, \cdots, n \text {. }
\end{aligned}
$$

By combining (2.2), (2.11) and (2.12) we get a system (of the form (2.2)) consisting of $m(n+2)$ equations in the $m(n+2)$ unknowns $u_{s}, \frac{\partial u_{s}}{\partial x_{h}}, \frac{\partial u_{s}}{\partial t}, s=1,2, \cdots, m, h=1,2, \cdots, n$.

THEOREM 4. Let $K:=(2 \delta)^{-1} n(n+2)^{2} m^{2}\left(\operatorname{Max}\left\{L_{1}, L_{2}\right\}\right)^{2}+2 m(n+2) \operatorname{Max}\left\{L_{0}, L_{1}\right\}$ and suppose that $u$ is a $C^{3,2}\left(\Omega \cup \partial_{t} \Omega\right) \cap C^{1}(\bar{\Omega})$ solution of $(2.2)$ and $\alpha$ is a positive $C^{2,1}\left(\Omega \cup \partial_{t} \Omega\right)$ function. Then the product

$$
\alpha(x, t)\left[|u(x, t)|^{2}+|\nabla u(x, t)|^{2}\right]=\alpha(x, t) \sum_{s=1}^{m}\left[\left|u_{s}\right|^{2}+\sum_{i=1}^{m}\left|\frac{\partial u_{s}}{\partial x_{i}}\right|^{2}+\left|\frac{\partial u_{s}}{\partial t}\right|^{2}\right]
$$

cannot attain a positive maximum at any point in $\Omega \cup \partial_{t} \Omega$ where $\alpha$ satisfies (2.4).

COROLLARY 5. Let $K$ be the same number of Theorem 4. Then, for any $C^{3,2}\left(\Omega \cup \partial_{t} \Omega\right) \cap C^{1}(\bar{\Omega})$ solution $u$ of the system (2.2), we have

$$
\|u\|_{0, \Omega}^{2}+\|\nabla u\|_{0, \Omega}^{2} \leq \exp (K T)\left(\|u\|_{0, \partial_{p} \Omega}^{2}+\|\nabla u\|_{0, a_{p} \Omega}^{2}\right)
$$

or equivalently,

$$
\|u\|_{1, \Omega} \leq \exp (K T / 2) \cdot\|u\|_{1, \partial_{p} \Omega} .
$$

REMARK. Under the condition that either $\left(c_{s j}\right)_{m \times m}$ is a constant matrix or $\left(c_{s,}\right)_{m \times m}$ is invertible for all $(x, t) \in \Omega$, the unknowns $u_{s}, s=1, \cdots, m$, can be eliminated from the system (2.2), (2.11), (2.12), and then a system of $m(n+1)$ equations in the gradient of $u$ yields a maximum principle for $\alpha|\nabla u|^{2}$.

ACKNOWLEDGEMENT. The author thanks Professor G.N. Hile and the anonymous referee for some helpful suggestions and comments.

\section{REFERENCES}

1. DOW, M.A., Strong maximum principles for weakly coupled systems of quasilinear parabolic inequalities, J. Austral. Math. Soc. 19 (1975), 103-120.

2. HILE, G.N. and PROTTER, M.H., Maximum principles for a class of first-order elliptical systems, J. Differential Equations 24 (1977), 136-151.

3. PROTTER, M.H. and WEINBERGER, H.F., Maximum Principles in Differential Equations, Springer-Verlag, Berlin, 1984.

4. STYS, T., On the unique solvability of the first Fourier problem for a parabolic system of linear differential equations of second order, Prace Mat. 9 (1965), 283-289.

5. WEINBERGER, H.F., Invariant sets for weakly coupled parabolic and elliptic systems, Rend. di Mat. 8 (1975), 295-310.

6. ZHOU, C., Maximum principles for weakly coupled parabolic systems, Appl. Analysis 47 (1992), 57-69. 


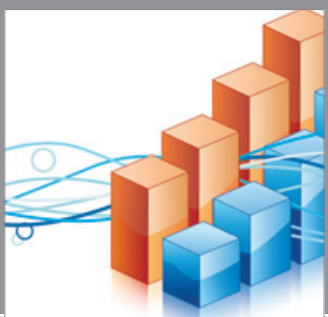

Advances in

Operations Research

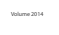

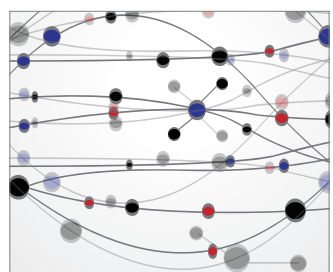

\section{The Scientific} World Journal
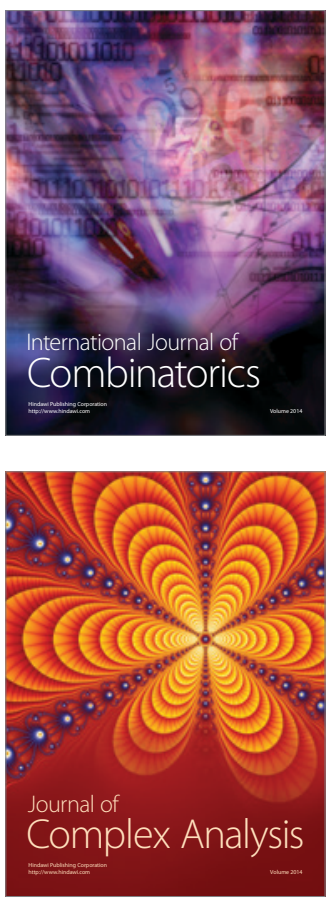

International Journal of

Mathematics and

Mathematical

Sciences
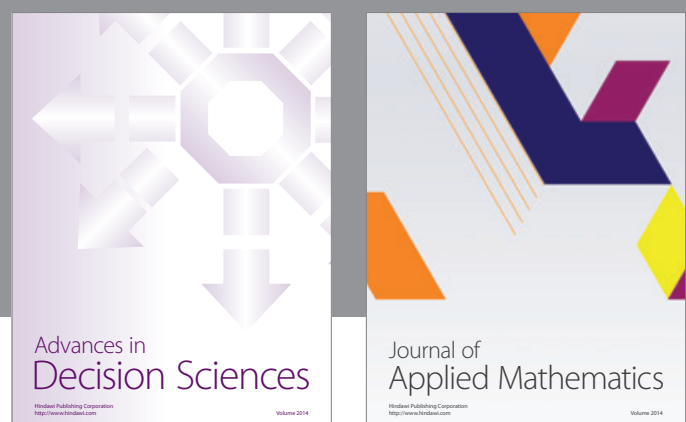

Journal of

Applied Mathematics
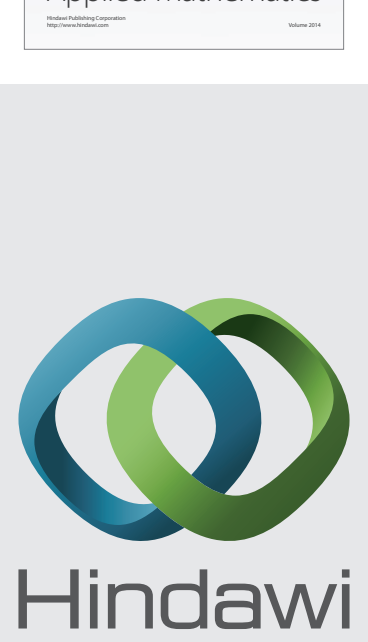

Submit your manuscripts at http://www.hindawi.com
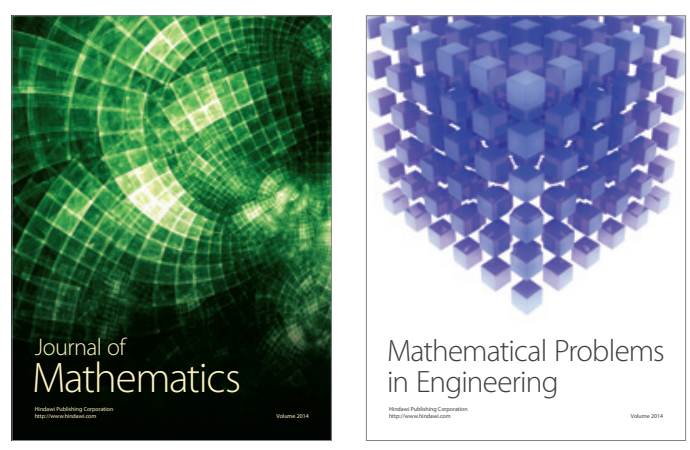

Mathematical Problems in Engineering
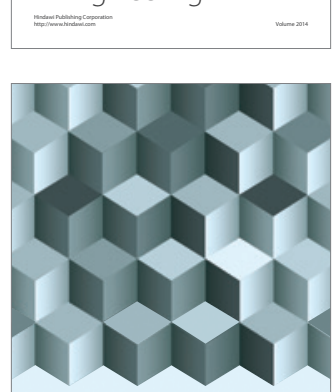

Journal of

Function Spaces
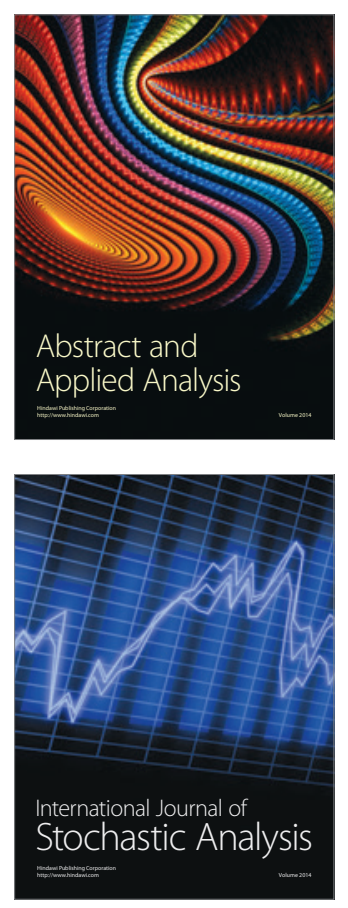

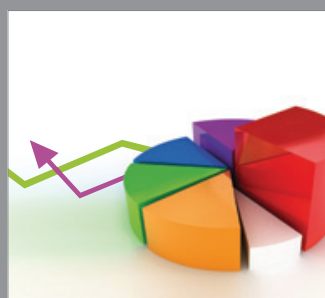

ournal of

Probability and Statistics

Promensencen
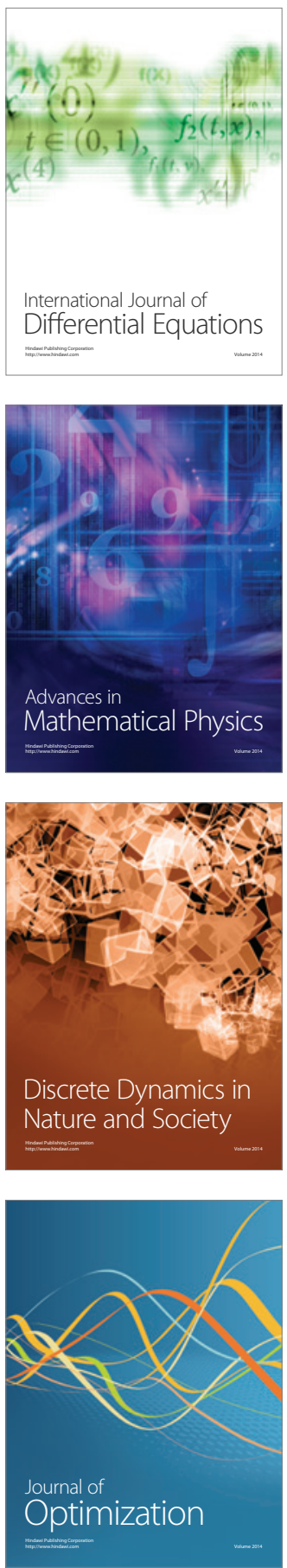